\title{
Study on combat network self-recovery mechanism based on load distribution
}

\author{
Ming-Xing Zhang, Guang-Quan Cheng, Shui-Fa Mei and Yang-He Feng \\ Science and Technology on Information Systems Engineering Laboratory, National \\ University of Defense Technology, Changsha, 410073, China \\ Troop 61833, China \\ Science and Technology on Information Systems Engineering Laboratory, National \\ University of Defense Technology, Changsha, 410073, China \\ E-mail: cgq299@163.com
}

\begin{abstract}
Under the combat-targeted network layer description, the paper establishes layered network model for combat-targeted networks so as to facilitate the study on its selfrecovery mechanism. Furthermore, it puts forward the optimal self-recovery strategy of the early warning network layer based on the load redistribution theory. Finally, simulation analysis is performed on the feasibility of the previously studied early warning network layer self-recovery mechanism via the simulated attack. The result shows that the target network's self-recovery mechanism can better utilize the combat capability of the remaining nodes while maintaining greater early warning capacity and minimizing the delay in each node type in the network.
\end{abstract}

Keywords: Target Network; Self-recovery Mechanism; Load Distribution.

\section{Description and Measurement Indicators Of The Combat-Targeted Network Layers}

\subsection{Early warning network layer}

The early warning network is a directed network $N^{(\text {radar })}\left(V^{(\text {radar })}, E^{(\text {radar })}\right)$ formed by the early warning nodes set $V^{(r a d a r)}$ and early warning network assistance connection edge set $E^{(\text {radar })}$. Node $i \in V^{(\text {radar })}$ represents the objects responsible for early warning tasks in the combat-targeted network. Early warning node $i$ has load $l_{i}^{(\text {radar })}$, delay and other properties. The load $l_{i}^{(\text {radar })}$ means the responsible early warning area of this early warning object $t$ in the current combat network, in which $0<l_{i}^{(\text {radar })}<l_{M A X}^{(\text {radar })}, l_{M A X}^{\text {(radar })}$ represents the largest early warning area that early warning equipment can cover.

Early-warning delay $d_{i}^{(\text {radar })}$ represents the average time from early warning and monitoring, attack detection to information processing. Early-warning delay 
$d_{i}^{(\text {radar })}$ is positively correlated with early-warning load $l_{i}^{(\text {radar })}$. The paper formulates a function of early-warning delay $d_{i}^{(\text {radar })}$ to early-warning $l_{i}^{(\text {radar })}$ to have the following Eq.(1):

$$
d_{i}^{(\text {radar })}=\alpha_{i}^{(\text {radar })}+\frac{\beta_{i}}{1+e^{\lambda_{i}-l_{i}^{(\text {radar })}}}
$$

In which $\alpha_{i}^{(\text {radar })}$ represents the shortest delay of the early warning node $\mathrm{i}$, and $\beta_{i}$ and $\lambda_{i}$ are parameters used to adjust the maximum delay and benchmark delay.

\subsection{Fire network layer}

Fire networks are directed networks $N^{(\text {fire })}\left(V^{(\text {fire })}, E^{(\text {fire })}\right)$ comprising fire nodes set $V^{(\text {fire })}$ and fire assistance connection edge set $E^{(\text {fire })}$. The node $i \in V^{(\text {fire })}$ represents the objects responsible for strike tasks in combat-targeted networks. The fire node $i$ has properties such as load $l_{i}^{(\text {fire })}$ and strike delay $d_{i}^{(f i r e)}$. The load of fire node $i l_{i}^{(f i r e)}$ represents the area covered by this fire object in the current combat network, in which $0<l_{i}^{(\text {fire })}<l_{M A X}^{(\text {fire })}, l_{M A X}^{(\text {fire })}$ represents the largest strike area which the fire object can reach by effective mobility and equipment ability. Strike delay $d_{i}^{(f i r e)}$ is positively correlated with fire load $l_{i}^{(\text {fire })}$. The paper formulates a function of strike delay $d_{i}^{(\text {fire })}$ to fire load $l_{i}^{(\text {fire })}$ to have the following Eq. (2)

$$
d_{i}^{(\text {fire })}=\alpha_{i}^{(\text {fire })}+\left(l_{i}^{(\text {fire })}\right)^{\gamma_{i}}
$$

In which $\alpha_{i}^{(\text {fire })}$ represents the shortest delay of fire node $i$ and $\gamma_{i}$ is the parameter used to adjust the sensitivity of delay to load.

\subsection{Command and control network layer}

The command and control network is the directed network $N^{(\mathrm{cmd})}\left(V^{(\mathrm{cmd})}, E^{(\mathrm{cmd})}\right)$ comprising command nodes set $V^{(\mathrm{cmd})}$ and communication connection edge set $E^{(\mathrm{cmd})}$. The node $i \quad i \in V^{(\mathrm{cmd})}$ represents the object responsible for the command and control tasks in the combat-targeted network. The command node $i$ $i \in V^{(\mathrm{cmd})}$ has the property of command and control delay $d_{i}^{(\mathrm{cmd})}$, which depends on the quantity of early warning nodes and fire node controlled by this node. The paper calculates the control nodes' delay $d_{i}^{(\mathrm{cmd})}$ according to Eq. (3).

$$
d_{i}^{(\text {cmd })}=\left(\sum_{(k, i) \in E^{(r-c)}} \frac{l_{k}^{(\text {radar })}}{\tau_{k}}\right)^{\rho}+\left(\sum_{(i, j) \in E^{(c-f)}} \kappa_{j}\right)^{\phi}
$$


In which $E^{(r-c)}$ represents the set from early warning nodes to command and control node's directed connection edge. $E^{(c-f)}$ represents the set from the command and control node to fire node's directed connection edge. $\tau_{k}$ represents the load-command \& control delay conversion parameter of earlywarning node $k . \kappa_{j}$ represents the average command and decision time required to command fire node $j . \rho$ and $\kappa$ are parameters respectively used to adjust the sensitivity of command and control to early warning information processing and command decision making.

The following four indexes are defined to measure the performance of combat-targeted network.

(1)Total early warning load $S l^{(\text {radar })}$

Total early warning load $S l^{(\text {radar })}$ is used to represent the total early warning area of the combat-targeted network. The computing method is show in Eq. (4). What is should be noted is that, if node $i$ fails, then $i \notin V^{(r a d a r)}$. Similar conclusions can be made with the nodes of other network layers, which will not be repeated the following text.

$$
S l^{(\text {radar })}=\sum_{i \in V^{\text {(radar })}} l_{i}^{(\text {radar })}
$$

(2) Average early warning delay $A d^{(\text {radar })}$

Average early warning delay $A d^{(\text {radar })}$ is used to represent the average delay time of early warning nodes in the combat-targeted network. The computing method is show in Eq.(5)

$$
A d^{(\text {cmd })}=\frac{\sum_{i \in V^{(c m d)}} d_{i}^{(c m d)}}{\left\|V^{(c m d)}\right\|}
$$

(3) Average command and control delay $A d^{(\mathrm{cmd})}$

Average command and control delay $A d^{(\mathrm{cmd})}$ is used to represent the average delay time of command and control nodes in the combat-targeted network. The computing method is show in Eq.(6).

$$
A d^{(c m d)}=\frac{\sum_{i \in V^{(c m d)}} d_{i}^{(c m d)}}{\left\|V^{(c m d)}\right\|}
$$

(4) OODA weighted link average delay $A d^{(r-c-f)}$

An OODA link is to spot the targets from an early warning node $i$ and then to report the information to the command and control node $k$, after which the command and control node gives order to fire node $j$ to strike the target. Every OODA link has a delay $d_{i j}^{(r-c-f)}$ and the $d_{i j}^{(r-c-f)}$ is calculated by Eq.(7): 


$$
d_{i j}^{(r-c-f)}=\sum_{(i, m) \in E^{(r-f)}} d_{i m}^{(r-f)}+\sum_{(n, j) \in E^{(f-c)}} d_{n j}^{(f-c)}+\tilde{d}_{\text {leader }(i), \operatorname{leader}(j)}^{(\mathrm{cmd})}
$$

In which leader (i) is the function for the number of the direct superior command and control node which returns to node i. $\tilde{d}_{m n}^{(\mathrm{cmd})}$ represents that, when the command and control is increased: a) $\tilde{d}_{m n}^{(c m d)}=d_{m n}^{(c m d)}$ if $(m, n) \in E^{(c m d)}$ or, b) if $(m, n) \notin E^{(\mathrm{cmd})}$, then following Eq. (8):

$$
\tilde{d}_{m n}^{(c m d)}=d_{m, \text { leader }(m)}^{(\mathrm{cm})}+d_{\text {leader }(n), n}^{(\mathrm{cmd})}+\tilde{d}_{\text {leader }(m), \text { leader }(n)}^{(\mathrm{cmd})}
$$

Specifically, $\tilde{d}_{m n}^{(c m d)}=0$ if $m=n$

As to a combat-targeted network, every OODA link is of a different significance. So, weight $w_{i j}^{(r-c-f)}$ should be given according to the real-world use frequency of every OODA link. And $\sum_{i \in E^{(\text {radar })}} \sum_{j \in E^{(\text {fre })}} w_{i j}^{(r-c-f)}=1$. The average delay weighted $A d^{(r-c-f)}$ of all the OODA links in the combat network is defined by (9).

$$
A d^{(r-c-f)}=\sum_{i \in E^{(r a d a r)}} \sum_{j \in E^{(f i r e)}} w_{i j}^{(r-c-f)} d_{i j}^{(r-c-f)}
$$

\section{Study on the self-recovery mechanism of early warning network layers}

The optimal self-recovery mechanism of the combat-targeted network is to launch the best self-recovery mechanism when the target network is under attack, so as to ensure that the network system can give full play to the combat capability of the remaining combat units. The paper utilizes layered optimization to explore the optimal self-recovery mechanism of combat-targeted network's early warning network layer under attack from the enemy's viewpoint.

\subsection{The local load redistribution method}

In conventional cascading network failure models ${ }^{[3]}$, when node $i$ fails after strike, the failed node's load will be transferred to its nearby node so as to ensure the effective operation of the whole network. As is shown in Figure $1, \Omega^{\text {(radar })}$ represents the combination of failure nodes in the early warning network. Therefore, the network's load will be totally redistributed due to the failure of the nodes, i.e. $l_{j}^{(\text {radar })}=l_{j}^{(\text {radar })}+\Delta l_{j}^{(\text {radar })}, j \in \Gamma_{i}^{(\text {radar })}$, in which $\Gamma_{i}^{(\text {radar })}$ is the combination of all the early warning assistance adjacent nodes. $l_{j}^{\prime(\text { radar })}$ is the load after the node $j$ load redistribution and $\Delta l_{j}^{(\text {radar })}$ is the load increment. Based 
on the load redistribution ${ }^{4,5}$, the paper puts forward the following methods.

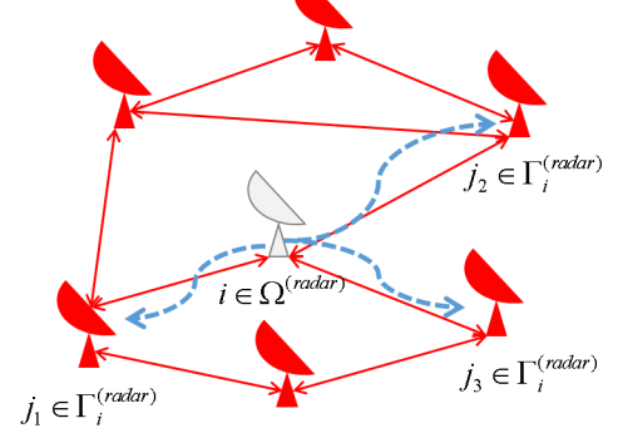

Fig. 1 The local load redistribution strategy

\subsection{The overall load redistribution method}

Different from the conventional local distribution, the paper puts forward a method of overall load redistribution, namely to adjust the load of the overall nodes when some early warning nodes in the network fail and to reduce $A d^{(\text {radar })}$ while ensuring the maximized $S l^{(\text {radar })}$. Radar Network Robustness Optimization in the first early warning network can be generalized as RNRO.

$$
\begin{gathered}
\max S l^{(\text {radar })}=\sum_{i \in V^{\text {(radar })}} l_{i}^{(\text {radar })} \\
\min A d^{(\text {radar })}=\frac{\sum_{i \in V^{(\text {radar })}} d_{i}^{(\text {radar })}}{\left\|V^{(\text {radar })}\right\|} \\
\Delta l_{j}^{(\text {radar })}=-\sum_{i \in \Gamma_{j}^{\text {(radar })}} t_{i j}^{(\text {radar })}, \forall j \in V^{(\text {radar })} \cup \Omega^{(\text {radar })} \\
\Delta l_{i}^{(\text {radar })} \leq l_{i}^{(\text {radar })}, \forall i \in V^{(\text {radar })} \cup \Omega^{(\text {radar })} \\
t_{i j}^{(\text {radar })} \leq c_{i j}^{(\text {radar })}, \forall i, j \in V^{(\text {radar })} \cup \Omega^{(\text {radar })} \\
t_{i j}^{(\text {radar })}+t_{j i}^{(\text {radar })}=0, \forall i, j \in V^{(\text {radar })} \cup \Omega^{(\text {radar })} \\
t_{i j}^{(\text {radar })} \in \square, \forall i, j \in V^{(\text {radar })} \cup \Omega^{(\text {radar })}
\end{gathered}
$$

In which $t_{i j}^{(\text {radar })}$, the decision variable for RNRO, represents the load from node $i$ to node $j . d_{i}^{\prime(\text { radar })}$ is the delay after the load redistribution of node $i$. The RNRO is a multi-target optimization issue.

As to a real-world combat-targeted network, delay of each reconnaissance node must be minimized when a large enough reconnaissance area is first ensured. So, in terms of the above-mentioned multi-target optimization issue, it 
is a multi-target optimization issue with target priority. The priority of the optimization target (8) is higher than optimization target(9).

\section{Appraisal of combat-targeted network self-recovery effects under the simulated attack experiment}

This section simulates the attack to a certain combat target and studies the response mechanism of the attacked subject to reduce the loss of the target network system function. Combat-targeted networks are under the same attack with conventional adjustment mechanisms (local load redistribution and substitute command methods) and mechanisms put forward in the paper (overall load balance, command and control and overall transfer methods). And network measurement of the two mechanisms under the same attack is inspected.

Self-recovery mechanisms of the whole combat-targeted network under different methods are inspected from four dimensions as the early warning node failure increases. Results are shown in Figure 2.


Fig. 2 The measurement changes of the network after the early warning nodes are attacked

From Figure 2(a), we can see that OODA weighted link average delay of combat-targeted network adjusted with these two methods basically increases first and then decreases as early warning nodes decrease. Because when early warning nodes just begin to decrease, the early warning load of failed nodes can be shared by other early warning nodes so as to prevent the overall early warning load from dramatically decreasing, according to Figure 2(c). But the 
cost is that early warning delay of the remaining early warning nodes will increase, according to Figure 2(d). Meanwhile, because early warning information is more concentrated in the remaining early warning nodes, early warning information is more intensively reported to certain nodes when early warning nodes are reported to command and control nodes, so as to increase the processing delay of these command and control nodes, thus putting OODA weighted link average delay on a rising phase. As the failed early warning nodes increase, the overall early warning load decreases sharply, thus greatly decreasing early warning information and gradually reducing OODA weighted link average delay. During the whole course of the gradual early warning node decrease, we can see the methods put forward in the paper feature shorter OODA weighted link average delay, average command and control delay, and average early warning delay than conventional methods, which effectively reduces the cost and fully reflects the superiority of the former.

\section{Conclusion}

To better understand the response strategies that the enemy combat-targeted network will take after being attacked, the paper carries out explorative study on the layered optimal self-recovery mechanism and established network models. It also establishes four dimensions of network performance measurement indicators so as to facilitate quantitative analysis on the combat-targeted network. Later, from the early warning network layer, it makes an analysis and study on the optimal self-recovery mechanism under attack, and carries out simulative attack experiment on the network data of a combat instance, and compares the different performances of conventional adjustment methods and those put forward in the paper. Theoretical analysis and simulation experiments all show that, compared with conventional methods, the methods put forward in the paper can better capitalize on the combat capability of the remaining nodes, thus minimizing the delay of various nodes in the network.

\section{Acknowledgments}

This paper was supported in part by National Natural Science Foundation of China under Grant No. 61201328, 71471175, Doctoral Fund of Ministry of Education of China under Grant No. 20114307120021 and Hunan Provincial Natural Science Foundation of China under Grant No. 13JJ4011. 


\section{References}

1. Callaway DS, Newman ME, Strogatz SH, Watts DJ. Network robustness and fragility: Percolation on random graphs[J]. Physical review letters. 2000, 85(25): 5468.

2. Cohen R, Erez K, Ben-Avraham D, Havlin S. Breakdown of the Internet under intentional attack[J]. Physical review letters. 2001, 86(16): 3682.

3. Lu Yongzhong. Analysis on the Vulnerability of Heterogeneous Networks Based on Cascading Failure Models [D]. Master's thesis, National University of Defense Technology, 2014.

4. Pham PP, Perreau S. Performance analysis of reactive shortest path and multipath routing mechanism with load balance[C] Proceedings of the INFOCOM 2003. Twenty-Second Annual Joint Conference of the IEEE Computer and Communications. IEEE Societies. 2003, 1: 251-259.

5. Zhou Yinglian, Liu Fu, Research on Server Load Balance Technology. Computer \& Digital Engineering. 2010, 38(4): 11-14. 